\title{
A Ptychographic Approach for Low Dose Electron Imaging of Organic Molecules
}

KAYLA NGUYEN ${ }^{1}$, Yi Jiang ${ }^{2}$, Blanka Janicek $^{3}$, Priti Kharel ${ }^{4}$ and Pinshane Huang ${ }^{4}$

${ }^{1}$ University of Illinois at Urbana-Champaign, URBANA, Illinois, United States, ${ }^{2}$ Advanced Photon Source, Argonne National Laboratory, Lemont, IL 60439, USA, United States, ${ }^{3}$ University of Illinois at Urbana-Champaign, Urbana, Illinois, United States, ${ }^{4}$ University of Illinois at Urbana-Champaign, United States

Real-space imaging at atomic or near-atomic resolution via transmission electron microscopy (TEM) has been invaluable for understanding the structure and bonding of inorganic materials and biological macromolecules, but high-resolution electron microscopy techniques are lacking for small organic molecules. While aberration-corrected electron microscopes can image every carbon atom in graphene with electron doses of $10^{4}-10^{6} \mathrm{e} / \AA^{2}$, organic molecules are extremely dose sensitive with dose limits of $0.5-12,000 \mathrm{e} / \AA^{2}[1]$. As a result, atomic-resolution imaging of carbon and other light elements in individual small molecules has largely remained out of reach. Here, we aim to push imaging resolution of dosesensitive molecular crystals using 4-dimensional scanning transmission electron microscopy (4D-STEM) and advanced ptychographic reconstruction algorithms (Fig. 1) [2,3]. Our work indicates that ptychographic reconstructions of monolayers of molecules could make it possible to solve atomic structures of small molecules directly through low-dose, real-space imaging.

Our approach utilizes recent developments in electron ptychography. Previous results using focused incident probes demonstrated that imaging resolution can be pushed beyond current annular dark field (ADF-STEM) capabilities [4]. Furthermore, improvements utilizing a mixed-state algorithm [5] with a defocused probe has produced successful reconstructions at extremely low doses of 375 electrons $/ \AA^{2}$ on inorganic 2D materials while maintaining better than $1.59 \AA$ resolution [6]. These results indicate that electron ptychography may also be a route for low-dose imaging of organic molecules. Using electron microscopy simulations, we conducted mixed-state ptychography reconstructions to determine the feasibility and limits of electron ptychography for organic molecules (Fig. 1a). As a test system, we simulated $\mathrm{CuPcCl}_{16}$, a planar organic molecule (Fig. 1b), on graphene (Fig. 1c).

In Figure 2, we compared simulated low-dose ADF images to mixed-state ptychography. We find that center $\mathrm{Cu}$ metal atom in the molecule becomes visible in the ADF data after averaging 10 images, while low-Z elements are still not visible even after averaging 100 ADF images (Fig. 2 a-c). At equivalent doses, mixed-state ptychography performs much better (Fig. 2 d-f): we are able to resolve the shape of the molecules and the low- $\mathrm{Z}$ carbon rings within the $\mathrm{CuPcCl}_{16}$ structure in (f) with 100 class-averaged images. Our data indicates that at the same electron dose, ptychography produces higher signal-to-noise ratio and structural information than ADF imaging, and that a resolution of 1.3-1.5 angstroms is feasible with realistic doses and acquisition conditions with signal averaging.

This work was supported by the NSF-MRSEC program under NSF Award Number DMR-1720633 and the L'Oreal For Women In Science Postdoctoral Fellowship. 

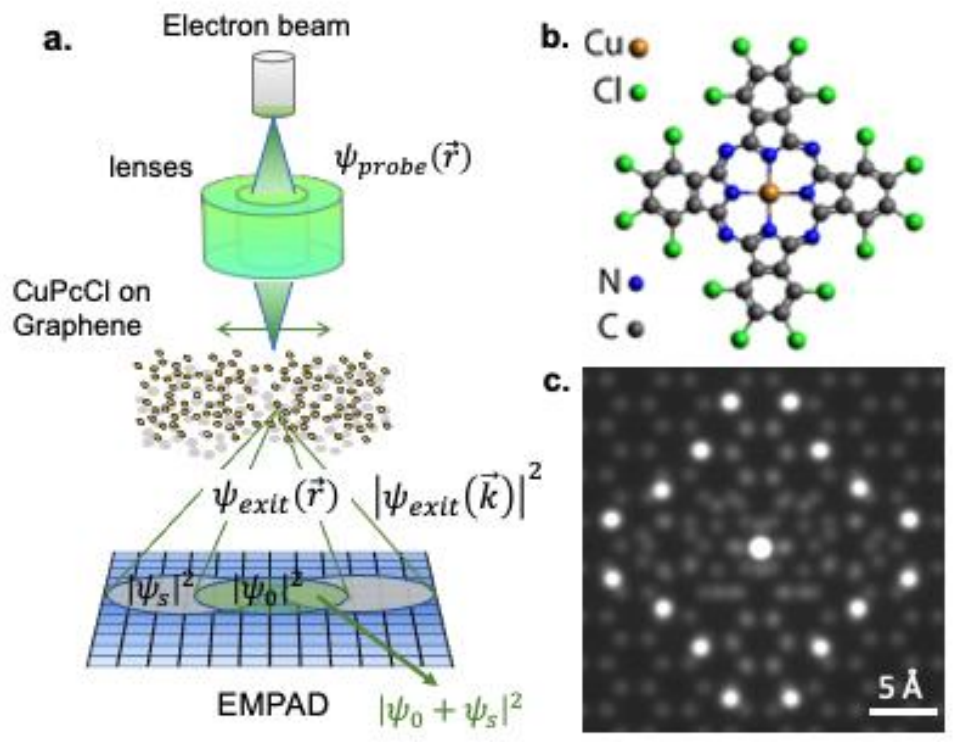

Figure 1. Figure 1. Schematic of 4D-STEM showing the molecular structure of b) CuPcCl16 and c) simulated object function of $\mathrm{CuPcCl16}$ on graphene used for ptychographic reconstructions.

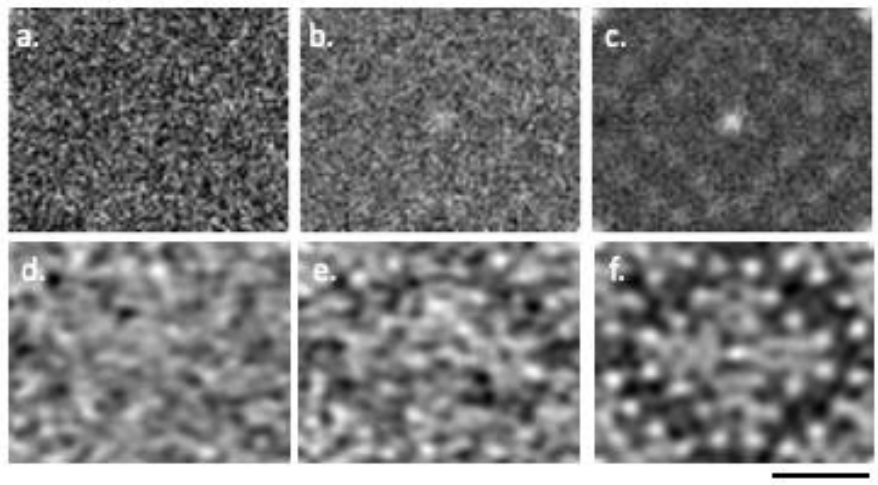

Figure 2. Figure 2. ADF and mixed-state ptychographic simulations of $\mathrm{CuPcCl} 16$ on graphene at electron dose of 800 electrons/2 at $300 \mathrm{kV}$ beam energy and $18 \mathrm{mrad}$ semi-convergence angle. Using ADFSTEM, we class average a) 1, b) 10, and c) $100 \mathrm{CuPcCl16}$ molecule(s) on graphene. We performed 
mixed-state ptychography and class averaged d) 1, e) 10 and f) 100 molecule(s). We find that mixed-state ptychography gives us information from the molecular shape and also its internal structure, whereas ADF only gives us the molecule's overall shape in (c) with 100 images class averaged. 10 scale bar in (f) is used for a-f.

\section{References}

[1] Egerton, R.F. Micron. 119, 72-87 (2019).

[2] Odstrcil, M. et al. Optics Express, 26, 3108-3123 (2018).

[3] Wakonig, K. et al. Journal of Applied Crystallography. 53, 574-584 (2020).

[4] Jiang, Y. et al. Nature 559, 343-349 (2018).

[5] Thibault, P. et al., Nature, 494, 68-71 (2013).

[6] Chen, Z. et al. Nature Comm. 11, 1-10 (2020).

[7] Mittelberger, A. et al., Scientific Reports 8, 1(2018.

[8] Kharel, P. et al., Microsc. Microanal. 26 (Suppl. 2), 2970-2971 (2020). 